ARTICLE

\title{
An in situ self-assembly template strategy for the preparation of hierarchical-pore metal-organic frameworks
}

Hongliang Huang ${ }^{1,2}$, Jian-Rong $\mathrm{Li}^{2}$, Keke Wang ${ }^{1}$, Tongtong Han ${ }^{1}$, Minman Tong ${ }^{1}$, Liangsha Li ${ }^{1}$, Yabo Xie ${ }^{2}$, Qingyuan Yang ${ }^{1}$, Dahuan Liu ${ }^{1} \&$ Chongli Zhong ${ }^{1}$

Metal-organic frameworks (MOFs) have recently emerged as a new type of nanoporous materials with tailorable structures and functions. Usually, MOFs have uniform pores smaller than $2 \mathrm{~nm}$ in size, limiting their practical applications in some cases. Although a few approaches have been adopted to prepare MOFs with larger pores, it is still challenging to synthesize hierarchical-pore MOFs (H-MOFs) with high structural controllability and good stability. Here we demonstrate a facile and versatile method, an in situ self-assembly template strategy for fabricating stable H-MOFs, in which multi-scale soluble and/or acid-sensitive metal-organic assembly (MOA) fragments form during the reactions between metal ions and organic ligands (to construct MOFs), and act as removable dynamic chemical templates. This general strategy was successfully used to prepare various $\mathrm{H}-\mathrm{MOFs}$ that show rich porous properties and potential applications, such as in large molecule adsorption. Notably, the mesopore sizes of the H-MOFs can be tuned by varying the amount of templates.

\footnotetext{
${ }^{1}$ State Key Laboratory of Organic-Inorganic Composites, Beijing University of Chemical Technology, Beijing 100029, China. ${ }^{2}$ Beijing Key Laboratory for Green Catalysis and Separation, Department of Chemistry and Chemical Engineering, College of Environmental and Energy Engineering, Beijing University of Technology, Pingleyuan 100, Chaoyang, Beijing 100124, China. Correspondence and requests for materials should be addressed to J.-R.L. (email: jrli@bjut.edu.cn) or to C.Z. (email: zhongcl@mail.buct.edu.cn).
} 
$\mathrm{P}$ orous materials have been attracting intense research interest due to their broad range of possible applications ${ }^{1}$. Tailoring structures and pore properties with rational design and controllability of porous materials are crucial for their specific applications, but challenging in practical preparation. Traditional porous solids such as zeolites, activated carbon and mesoporous silica are relatively difficult in modifying and tailoring their structures and functions, in particular at the molecular level, whereas newly developed metal-organic frameworks (MOFs), composed of organic linkers and inorganic nodes, are recognized to be easy in this respect ${ }^{2,3}$. This type of materials has shown great potential in various applications including adsorption, separation, catalysis, sensing and so on $^{4-6}$. So far, the related research mainly focuses on the microporous MOFs. In particular, some stable microporous MOFs were reported in recent years ${ }^{7-9}$, which greatly promoted the practical applications of these new materials. The small pore size in microporous MOFs benefits the adsorption and separation of small molecules, but restricts their diffusion and also prevents larger molecules from accessing the MOF channels, thus greatly limiting their applications in some cases ${ }^{10}$. Thereby, the design and preparation of MOF materials with larger pore sizes are imperative, yet challenging to date.

Two approaches have mainly been developed to 'enlarge' pores of MOFs: (i) construct MOFs by using large building units (metal clusters and/or organic ligands) ${ }^{19-14}$ and (ii) fabricate MOF materials with large pores as crystal 'defect' 10,15 . With respect to the former, the ligand-extension strategy was widely adopted $^{16-19}$. However, the pore size in the resulting periodic nanostructures of MOFs is still limited to be smaller than $10 \mathrm{~nm}$ (ref. 17). In particular, with the increase of pore size, the frameworks usually become unstable in most cases. On the other hand, the ligand extension normally also results in the interpenetration of the structures, which would dramatically decrease the pore size $e^{16,17}$. Therefore, increasing the pore size of MOFs and keeping their framework stable remain a great challenge in design and synthesis. In addition, from the viewpoint of cost, synthesis of large linkers is too expensive for practical applications of resulting MOF materials. For the latter approach, the ligands can be cheap but the fabrication methods are pivotal and difficult to follow in most cases.

Alternatively, template methods have been explored to prepare stable hierarchical-pore MOFs (H-MOFs) containing both micropores and mesopores/macropores ${ }^{20}$. As we know that the hard template and the soft template methods have been widely adopted in preparing mesoporous and other hierarchical-pore materials. For the hard template method, as calcinations or acid etchants are often required to remove the templates ${ }^{21}$, MOFs can hardly be kept stable during this process, thus limiting its application in fabricating H-MOFs. For the soft template method, surfactants or block copolymers are used as templates, being easily manipulated and feasible for some MOFs, and thus has been used in preparing some $\mathrm{H}-\mathrm{MOFs}^{20,22-26}$. For example, cetyltrimethylammonium bromide has been used as the template to prepare hierarchical-pore $\mathrm{Cu}_{3}(\mathrm{BTC})_{2} \quad\left(\mathrm{H}_{3} \mathrm{BTC}=1,3,5\right.$ benzenetricarboxylic acid) in water-ethanol or ionic liquid systems ${ }^{20,23-25}$. Similarly, triblock copolymers such as P123 and F127, which are widely used to prepare meso-porous silicon, have also been used to prepare $\mathrm{H}-\mathrm{MOFs}^{27-31}$. However, as most MOFs were synthesized in polar solvents, such as $\mathrm{N}, \mathrm{N}$ dimethylformamide (DMF), N,N-dimethylacetamide and $\mathrm{N}, \mathrm{N}$ diethylformamide, traditional surfactants are not able to play the role of a template due to their amphipathy. Therefore, the soft template method also has a limitation in preparing H-MOFs. Other methods for preparing H-MOFs by crystal 'defect' include gelation $^{32}, \mathrm{CO}_{2}$-expanded liquids ${ }^{33}$, pseudomorphic replication by transformation of oxide ${ }^{34}$ and so on. However, these innovative approaches are complicated to operate, being comparatively difficult to extend to other MOFs.

For the template method, it is crucial to select appropriate template in the preparation of porous materials. The template should not only have a good interaction/affinity with reaction precursors but also should be able to remove easily, keeping the structure of targeted porous materials intact. In addition, for some practical applications, bad stability of a material often is viewed as extremely negative. However, the weakness of the instability in a material can also be a positive factor in some cases $^{35}$. Motivated by the context described above, herein we propose to use metal-organic assemblies (MOAs) including MOFs as the templates to prepare $\mathrm{H}-\mathrm{MOF}$ through an in situ self-assembly approach, where both the targeted porous materials and the templates belong to coordination complexes, being compatible in structural nature and reaction activity. Simultaneously, we can take advantage of their relatively different stabilities to get desired H-MOFs, that is, unstable MOAs as templates and stable MOFs as parents for targeting H-MOFs.

It has been well-documented that although MOF-5 is stable in several solvents ${ }^{16}$, the sensitivity towards moisture and acid can lead to its structural collapse and decomposition ${ }^{36,37}$. In contrast, some MOFs are chemically, thermally and mechanically stable, such as $\mathrm{UiO}-66(\mathrm{Zr})^{38}$, which can maintain its crystal structure even in an acid solution. These differences in the stability stimulate us to try to use water- or acid-sensitive MOFs, such as MOF-5 as the potential template to synthesize stable $\mathrm{H}-\mathrm{UiO}$ $66(\mathrm{Zr})$.

Here we suppose that if this hypothesis can be accomplished, a lot of labile MOAs could be used as template sources for the preparation of various stable H-MOFs. Theoretically, in a selfassembly reaction process, the reversibility of coordination bonds in these coordination complexes could keep the MOAs template forming and disappearing during the stable MOFs forming and growing. Thus, the MOAs could indeed act as a chemical dynamic template to direct the construction of H-MOFs. In this work, we demonstrate this idea, a new template-based strategy, to prepare stable $\mathrm{H}$-MOFs by adopting an in situ self-assembly synthesis method (Fig. 1).

\section{Results}

Preparation of H-MOFs through two-step reactions. A series of proof-of-concept experiments were performed. First, we used MOF-5 (Fig. 2a) as a template precursor to prepare $\mathrm{H}-\mathrm{UiO}$ $66(\mathrm{Zr})$ (see Fig. 2d for the structure of UiO-66(Zr)) through a two-step reaction process. Nano-sized MOF-5 particles were first synthesized and mixed with $\mathrm{ZrCl}_{4}$ and terephthalic acid in DMF. The mixture was then heated under solvothermal reaction condition, similar to that for synthesizing UiO-66(Zr). After the reaction, the resulting product template@H-UiO-66 $(\mathrm{Zr})$ was washed with acid aqueous solution to get targeted material $\mathrm{H}-\mathrm{UiO}-66(\mathrm{Zr})$. Powder X-ray diffraction (PXRD) measurements show that the resulting $\mathrm{H}-\mathrm{UiO}-66(\mathrm{Zr})$ has the same diffraction patterns as the parent UiO-66(Zr) (Fig. 2e and Supplementary Fig.18). The $\mathrm{N}_{2}$ adsorption at $77 \mathrm{~K}$ demonstrates the formation of the hierarchical-pore material with both micropores and mesopores (Supplementary Fig. 5). The detail of the preparation process can be seen in the Supplementary Methods section.

Considering such a fact that the mesopore size (about $11 \mathrm{~nm}$ ) of the $\mathrm{H}-\mathrm{UiO}-66(\mathrm{Zr})$ is much smaller than that of originally added MOF-5 particles with the size in the range of $370 \sim 520 \mathrm{~nm}$ (Supplementary Figs 1 and 2, the evaluated polydispersity index is 0.1 ), we suspect that there exists a 'decomposition and/or rearrangement' of MOF-5 particles in the reaction system. 
a

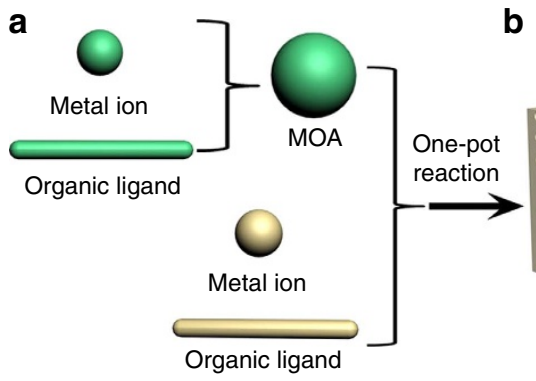

b

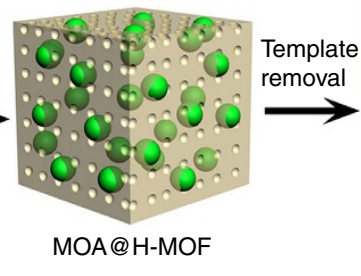

c

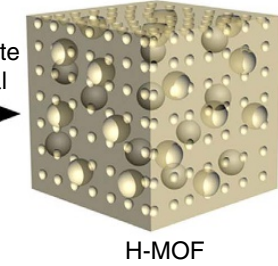

Figure 1 | Schematic representation for the preparation of H-MOF. (a) In situ self-assembly of MOA through the reaction between metal ion and organic ligand. (b) MOA@H-MOF composite formed by one-pot self-assembly reaction. (c) H-MOF formed through removing MOA template.

a

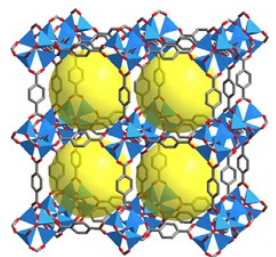

d

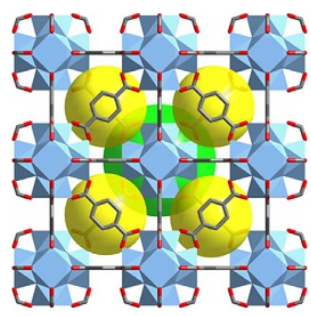

g

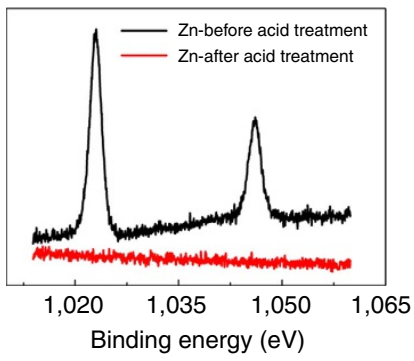

b

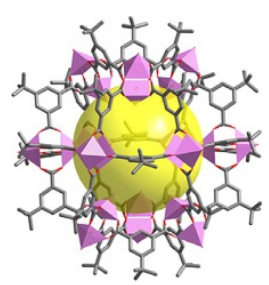

e

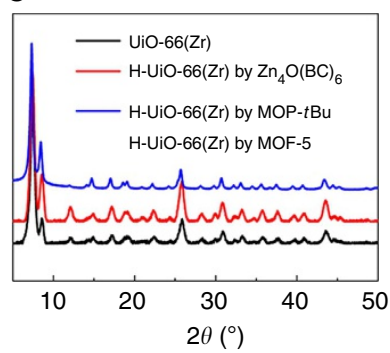

h

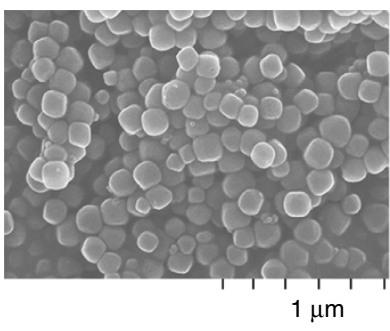

C

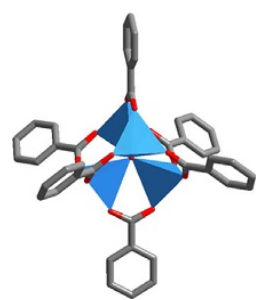

f

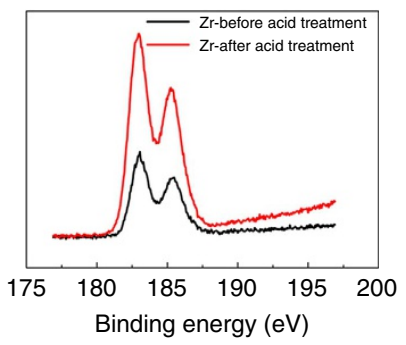

i

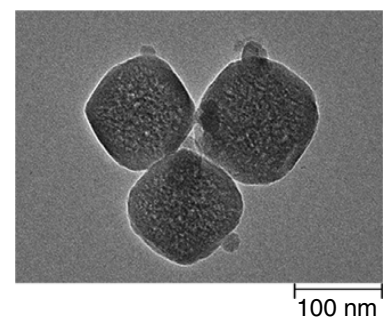

Figure 2 | Template-based preparation and characterization of H-UiO-66(Zr). (a) The structure of MOF-5, (b) the structure of metal-organic polyhedron (MOP-tBu), (c) the structure of $\mathrm{Zn}_{4} \mathrm{O}(\mathrm{BC})_{6}$ and (d) the structure of $\mathrm{UiO}-66(\mathrm{Zr})$. Colour scheme: $\mathrm{Zn}$ atom, light blue polyhedron; $\mathrm{Cu}$ atom, pink polyhedron; $\mathrm{Zr}$ atom, cinerous polyhedron; $\mathrm{C}$ atom, grey; and $\mathrm{O}$ atom, red. All $\mathrm{H}$ atoms have been omitted for clarity. The yellow and green spheres represent the void inside of MOF and MOP. (e) PXRD patterns of $\mathrm{H}-\mathrm{UiO}-66(\mathrm{Zr}$ ) prepared using different template precursors. (f,g) The XPS spectra of template@H-UiO-66( $\mathrm{Zr}$ ) and $\mathrm{H}-\mathrm{UiO}-66(\mathrm{Zr})$ prepared with MOF-5 as the template precursor over the $\mathrm{Zr} 3 d$ and $\mathrm{Zn} 2 p$ spectral regions, respectively. (h) SEM image and (i) transmission electron microscope image of $\mathrm{H}-\mathrm{UiO}-66(\mathrm{Zr}$ ) prepared with MOF-5 as the template precursor.

This is indeed coincident with the characteristic of MOF-5 as a typical coordination complex, where MOF-5 particles were in a self-assembly stage of dynamic equilibrium due to the reversibility of the involved coordination bonds $s^{39,40}$. The general elemental analysis (EA) and inductive-coupled plasma (ICP) emission spectra analysis for the template@H-UiO-66 $(\mathrm{Zr})$ gave the contents of $\mathrm{Zr} 27.14, \mathrm{Zn} 11.05, \mathrm{C} 35.65 \%$ and $\mathrm{H} 1.57 \%$; however, for $\mathrm{H}-\mathrm{UiO}-66(\mathrm{Zr})$ they are $\mathrm{Zr} 31.24$, $\mathrm{Zn} 0.24$, C $33.49 \%$ and $\mathrm{H} 2.27 \%$, respectively. The mole ratio of $\mathrm{C} / \mathrm{Zn}$ in the 'template' was estimated (by assuming that all the $\mathrm{Zr}$ comes from $\mathrm{H}-\mathrm{UiO}-66(\mathrm{Zr})$ and $\mathrm{H}-\mathrm{UiO}-66(\mathrm{Zr})$ has the same element contents as that parent $\mathrm{UiO}-66(\mathrm{Zr}))$ to be 3.5 , being smaller than that in MOF-5 (theoretical value is 6) (Supplementary Table 2). This result indicates that in the formation of $\mathrm{H}-\mathrm{UiO}-66(\mathrm{Zr})$, the actual template is not MOF-5 particles but is some newly in situregenerated MOAs. This judgment can also be supported by the fact that no diffraction peaks of the MOF-5 were observed in template@H-UiO-66(Zr) (Supplementary Fig. 19). Besides, it was also found that the MOF-5 particles decompose within $1 \mathrm{~h}$ in the $\mathrm{ZrCl}_{4}$ DMF solution even at room temperature, which further reveals the 'decomposition and/or rearrangement' of MOF-5 in the reaction system. On the basis of these observations, the fact of 
a complicated self-assembly process could be thus justified. These generated MOAs act thus as the 'templates' to direct the formation of template@UiO-66( $\mathrm{Zr})$. During acid treatment, the 'template' was removed. Therefore, the acid treatment indeed acts as an activation process to clear the pores of the H-MOF.

As shown in Fig. 2f,g, X-ray photoelectron spectroscopy (XPS) spectra demonstrate that the template@H-UiO-66(Zr) sample contains both $\mathrm{Zn}$ and $\mathrm{Zr}$, but after acid treatment almost no $\mathrm{Zn}$ was detected (on the sample surface), while $\mathrm{Zr}$ remained. Based on the ICP analysis, a little bit $\mathrm{Zn}$ was identified in the $\mathrm{H}-\mathrm{UiO}$ $66(\mathrm{Zr})$, which might be attributed to the $\mathrm{Zn}$ ions anchoring (through coordinating with some groups, such as carboxylate) in mesopore surfaces of $\mathrm{H}-\mathrm{UiO}-66(\mathrm{Zr})$. These coordination groups were generated from the 'defect' of the UiO-66(Zr) framework. In addition, as given above, the $\mathrm{C}$ and $\mathrm{H}$ contents in $\mathrm{H}-\mathrm{UiO}-66(\mathrm{Zr})$ were close to the theoretical values ( $\mathrm{C} 34.6 \%$ and $\mathrm{H} 1.68 \%$ ) of parent $\mathrm{UiO}-66(\mathrm{Zr})$. Moreover, the Fourier transform infrared (FT-IR) spectrum indicates no free terephthalic acid remained in the pores of $\mathrm{H}-\mathrm{UiO}-66(\mathrm{Zr})$ (Supplementary Fig. 31).

Furthermore, high-angle annular dark-field scanning transmission electron microscopy (HAADF-STEM) analysis combined with energy dispersive X-ray spectroscopy (EDX) mapping reveals that $\mathrm{Zr}, \mathrm{O}$ and $\mathrm{C}$ elements were homogeneously distributed in the whole template@H-UiO-66(Zr) sample but $\mathrm{Zn}$ element was heterogeneous (Supplementary Fig. 40). In addition, scanning electron microscope (SEM) and transmission electron microscope images show that the H-UiO-66(Zr) sample is of small nanoparticles $(\sim 100 \mathrm{~nm})$ and has a sponge-like morphology, which implies the existence of crystal defect (Fig. 2h,i). These results suggest the existence of irregularly distributed mesopores in $\mathrm{H}-\mathrm{UiO}-66(\mathrm{Zr})$. All these results confirm the existence of the $\mathrm{Zn}$-based templates in template@H-UiO$66(\mathrm{Zr})$ and these templates can be almost completely removed by the acid treatment to give the H-MOF material.

In addition, there exist hysteresis loops in the $\mathrm{N}_{2}$ adsorption isotherms of template@H-UiO-66(Zr) and $\mathrm{H}-\mathrm{UiO}-66(\mathrm{Zr})$, being indicative of mesoporous structures of both materials (Supplementary Fig. 6). Before the acid treatment, the mesoporous structure may be created by removal of part of soluble template fragments in the sample treatment process through the DMF washing. However, the surface area and pore volume are low in template@H-UiO-66(Zr). After acid treatment, the material became clearly more porous and the mesopore was much larger than that before treatment. This result also implies that the acid treatment indeed is just an activation process to clear the pores as discussed above. It should also be pointed out that although $\mathrm{UiO}-66(\mathrm{Zr})$ is not stable in phosphoric acid solution $^{41}$, it is stable in diluted $\mathrm{HCl}$ solution $^{38}$. As shown in Supplementary Fig. 8, $\mathrm{N}_{2}$ adsorption isotherms indicate no mesopore in $\mathrm{UiO}-66(\mathrm{Zr})$ after soaking in diluted $\mathrm{HCl}$ solution (pH 1) for $12 \mathrm{~h}$.

To further confirm the associated self-assembly process in the preparation of $\mathrm{H}-\mathrm{MOF}$ using this new method and its universal accessibility, another two types of MOAs were also chosen as the template precursors to carry out the synthesis experiments, including simple complex molecules and metal-organic polyhedra (MOPs) molecules. These molecule-based MOAs can be used as the supramolecular building units in constructing MOFs ${ }^{16,42,43}$. For the former, we explored $\mathrm{Zn}_{4} \mathrm{O}(\mathrm{BC})_{6}$ (BC, benzenecarboxylate; Fig. 2c) to again prepare $\mathrm{H}-\mathrm{UiO}-66(\mathrm{Zr}) . \mathrm{Zn}_{4} \mathrm{O}(\mathrm{BC})_{6}$ is a primary coordination complex about $2 \mathrm{~nm}$ in size, which is the secondary building unit of MOF-5 structure. Specifically, the $\mathrm{Zn}_{4} \mathrm{O}(\mathrm{BC})_{6}$ was first prepared by the reaction of $\mathrm{Zn}\left(\mathrm{NO}_{3}\right)_{2} \cdot 6 \mathrm{H}_{2} \mathrm{O}$ and benzoic acid in DMF. After the reaction, the resulting solution was cooled down to room temperature. Then, $\mathrm{ZrCl}_{4}$ and terephthalic acid were added and the mixture was heated under reaction conditions similar to those for the synthesis of parent UiO-66(Zr). Figure 2e and Supplementary Fig. 16 show that the resulting material also has the same PXRD pattern as that of the parent $\mathrm{UiO}-66(\mathrm{Zr})$. After washing with acid, the resulting $\mathrm{H}-\mathrm{UiO}-66(\mathrm{Zr})$ was harvested as confirmed by the PXRD, XPS spectra, ICP, EA, thermal gravimetric analysis (TGA) and $\mathrm{N}_{2}$ adsorption/desorption isotherms (Supplementary Figs 3,16,24 and 36). XPS spectrum indicates that almost no $\mathrm{Zn}$ exists in the $\mathrm{H}-\mathrm{UiO}-66(\mathrm{Zr})$ sample surface after acid treatment. The EA and ICP analyses gave the contents of $\mathrm{Zr} 27.95, \mathrm{Zn} \mathrm{12.75,} \mathrm{C} 45.32$ and $\mathrm{H} 3.15 \%$ in the template@H-UiO-66(Zr); however, for $\mathrm{H}-\mathrm{UiO}-$ 66(Zr) they are $\mathrm{Zr} 31.08, \mathrm{Zn} 0.25, \mathrm{C} 33.12$ and $\mathrm{H} 2.05 \%$. The evaluated mole ratio of $\mathrm{C} / \mathrm{Zn}$ of the 'template' in template@H$\mathrm{UiO}-66(\mathrm{Zr})$ is 6.8 , which is again much smaller than that in $\mathrm{Zn}_{4} \mathrm{O}(\mathrm{BC})_{6}$ molecule (theoretically, the mole ratio of $\mathrm{C} / \mathrm{Zn}$ is 10.5 ). This result indicates that there is a 'decomposition and/or rearrangement' of the $\mathrm{Zn}_{4} \mathrm{O}(\mathrm{BC})_{6}$ molecule in the in situ selfassembly reaction, the $\mathrm{Zn}_{4} \mathrm{O}(\mathrm{BC})_{6}$ is not the true template. In addition, as given above the $\mathrm{C}$ and $\mathrm{H}$ contents in $\mathrm{H}-\mathrm{UiO}-66(\mathrm{Zr})$ close to the theoretical value (C 34.6 and $\mathrm{H} 1.68 \%$ ) of parent $\mathrm{UiO}$ $66(\mathrm{Zr})$, suggesting the removal of most templates during the acid treatment. Moreover, again there is a little $\mathrm{Zn}$ in generated $\mathrm{H}-\mathrm{UiO}$ $66(\mathrm{Zr})$, probably due to the same reason as proposed above. On the other hand, HAADF-STEM image and EDX mapping also show that $\mathrm{Zr}, \mathrm{C}$ and $\mathrm{O}$ elements were homogeneously distributed in the whole template@H-UiO-66(Zr) but $\mathrm{Zn}$ element was heterogeneous (Supplementary Fig. 41).

To further explore the components of the molecule-based MOA templates in fabricating $\mathrm{H}-\mathrm{UiO}-66(\mathrm{Zr})$ and the template removability, we prepared the $\mathrm{H}-\mathrm{UiO}-66(\mathrm{Zr})$ by using $\mathrm{Zn}_{4} \mathrm{O}$ $\left(\mathrm{BC}-\mathrm{CH}_{3}\right)_{6} \quad\left(\mathrm{BC}-\mathrm{CH}_{3}\right.$, 4-methylbenzenecarboxylate) template precursor. As shown in Supplementary Fig. 38, based on the chemical shift at 20 p.p.m. the ${ }^{13} \mathrm{C}-\mathrm{NMR}$ spectrum indicates the existence of the $\mathrm{BC}-\mathrm{CH}_{3}$ in template@H-UiO-66( $\left.\mathrm{Zr}\right)$. After the acid treatment, this peak disappeared, indicating the removal of the template (Supplementary Fig. 39). Similarly, we also prepared the $\mathrm{H}$-UiO-66(Zr) by using $\mathrm{Zn}_{4} \mathrm{O}\left(\mathrm{BC}-\mathrm{NO}_{2}\right)_{6} \quad\left(\mathrm{BC}-\mathrm{NO}_{2}\right.$, 4nitrobenzoic acid). As shown in Supplementary Fig. 32, based on the wavenumber at 1,522 and $1,437 \mathrm{~cm}^{-1}$, the FT-IR spectrum indicates the existence of the $\mathrm{BC}-\mathrm{NO}_{2}$ in template@H-UiO$66(\mathrm{Zr})$. However, after the acid treatment these peaks disappeared, indicating the removal of the template. In terms of the above analysis, it was demonstrated that both metal ions and organic portions in template can be efficiently removed by the acid treatment, making sure the purity of resulting $\mathrm{H}$-UiO$66(\mathrm{Zr})$.

In the case of MOP- $t \mathrm{Bu}$ (Fig. 2b $)^{44}$, experimentally, assynthesized MOP- $t \mathrm{Bu}, \mathrm{ZrCl}_{4}$ and terephthalic acid were mixed and dispersed in DMF. The mixture solution was heated to address the reaction under the conditions similar to those for preparing parent $\mathrm{UiO}-66(\mathrm{Zr})$. Again, the resulting material has the same PXRD patterns as the $\mathrm{UiO}-66(\mathrm{Zr})$ and acid-treated sample exhibits the characteristic of the H-UiO-66(Zr) (Fig. 2e, Supplementary Figs 4,17,25 and 42). These results indicate that the MOPs can also act as the template precursors to construct H-MOFs.

Above proof-of-concept experiments reveal that MOAs with different original structural complexity and size, including primary complex molecule, supramolecular MOPs and structurally extended MOFs all can act as the template precursors for fabricating $\mathrm{H}-\mathrm{MOF}$. Interestingly, the pore size of resulting $\mathrm{H}-\mathrm{MOF}$ is independent on the size of these initially used MOAs or their particles, suggesting that there exists a 'decomposition and/or rearrangement' of them to form new MOAs, which act as the in situ self-assembly templates in the construction of these $\mathrm{H}-\mathrm{MOF}$ in given reaction systems. 
Preparation of H-MOFs through one-pot reaction. On the base of above interesting findings, we also tried to prepare $\mathrm{H}-\mathrm{MOFs}$ by a one-pot reaction approach, so as to further confirm the in situ self-assembly template mechanism, again on $\mathrm{H}-\mathrm{UiO}-66(\mathrm{Zr})$. Onepot reaction of $\mathrm{Zn}\left(\mathrm{NO}_{3}\right)_{2} \cdot 6 \mathrm{H}_{2} \mathrm{O}$, excess terephthalic acid and $\mathrm{ZrCl}_{4}$ in DMF was conducted under solvothermal conditions similar to those for the synthesis of parent UiO-66(Zr). After acid washing, PXRD and $\mathrm{N}_{2}$ adsorption confirm that the resulting material is $\mathrm{H}-\mathrm{UiO}-66(\mathrm{Zr})$ (Supplementary Figs 12a and 22a). With the same route, we also checked the applicability of this strategy in using $\mathrm{Zn}_{4} \mathrm{O}(\mathrm{BC})_{6}$ and MOP- $t \mathrm{Bu}$ precursors as template sources in one-pot reaction. The product based on $\mathrm{Zn}_{4} \mathrm{O}(\mathrm{BC})_{6}$ precursors was obtained from the reaction of $\mathrm{Zn}\left(\mathrm{NO}_{3}\right)_{2} \cdot 6 \mathrm{H}_{2} \mathrm{O}$, benzoic acid, $\mathrm{ZrCl}_{4}$ and terephthalic acid in DMF. After washing with acid, $\mathrm{H}-\mathrm{UiO}-66(\mathrm{Zr})$ was obtained as confirmed by the PXRD and $\mathrm{N}_{2}$ adsorption (Supplementary Figs 10a and 20a). It should be pointed out that the ligand exchange between formed MOFs and free ligands could exist in the solution of this system such as observed in UiO-66, Materials of Institute Lavoisier (MILs) and zeolitic imidazolate frameworks (ZIFs) systems ${ }^{45,46}$. Indeed, the UiO-66(Zr) with ligand missinglinker defects can also be prepared by adding given amount of modulators such as acetic acid, benzoic acid and trifluoroacetic acid in the reaction system ${ }^{47-49}$. However, the resulting new pores in these 'defect' UiO-66(Zr) was small and no obvious hysteresis loop was observed in their $\mathrm{N}_{2}$ adsorption-desorption isotherms. In addition, we also tried to prepare $\mathrm{H}-\mathrm{UiO}-66(\mathrm{Zr})$ with only adding $\mathrm{HBC}$ in the reaction system through the one-pot reaction between terephthalic acid and $\mathrm{ZrCl}_{4} \cdot \mathrm{N}_{2}$ adsorption indicates that no mesopore was generated in resulting material (Supplementary Fig. 7). Similarly, H-UiO-66(Zr) can also be prepared through the reaction of $\mathrm{Cu}\left(\mathrm{NO}_{3}\right)_{2} \cdot 3 \mathrm{H}_{2} \mathrm{O}, 5$ - $t$-butyl-1,3benzenedicarboxylic acid, $\mathrm{ZrCl}_{4}$ and terephthalic acid in DMF, followed by acid washing (Supplementary Figs 11a and 21a). All these results demonstrate that a one-pot reaction of MOA template precursors and targeted $\mathrm{H}-\mathrm{MOF}$ precursors is also feasible in the preparation of H-MOFs. As we expected, the 'formation and degradation/rearrangement' of the MOAs in the in situ one-pot reaction enable them to play a template role in the formation of mesoporous structures of resulting H-MOFs.

To examine the universality of this in situ self-assembly method, we performed additional synthesis experiments on a couple of other typical MOFs with good physicochemical stabilities, including ZIF-8, MIL-101(Cr), DUT-5 and several functionalized $\mathrm{UiO}-66(\mathrm{Zr})$ by one-pot reaction. As expected, 19 H-MOFs were successfully prepared and characterized by EA, ICP, PXRD, FT-IR, TGA and $\mathrm{N}_{2}$ adsorption (see Supplementary Information). Some structural features of eight representative H-MOFs are listed in Table 1 and the complete data are provided in Supplementary Table 1.

As a whole, it was found that in the preparation, actual templates can not be identified in all cases. Combining element analysis and infrared characterizations, we found that almost no organic residues from the template or free ligands were left in pores of resulting $\mathrm{H}-\mathrm{MOFs}$ after the acid treatment. It was also demonstrated that the crystallinity of all H-MOFs become to be bad compared with their parent MOFs, probably due to generating numerous defects in the structures of H-MOFs. The lost of crystallinity of H-MOFs in some cases has also been confirmed in literatures 28,32 . Furthermore, the crystallinity and the porosity are directly related to the template amounts as discussed in detail below. These H-MOFs have also a better purity, even if incomparable with their parent MOFs in some cases. For TGA results, some as-synthesized samples present several weight-loss steps, which could be basically ascribed to (1) the loss of a small quantity of free guest solvent molecules in pores of $\mathrm{H}$-MOFs at lower temperature range, (2) the lose of high boiling point solvent molecules and coordinated water molecules in the $\mathrm{H}-\mathrm{MOFs}$ structures at higher temperature range, and (3) the decomposition of the H-MOFs frameworks. The second step usually was combined by the third, representing a sequential weight loss in a broad temperature range. It was also found that TGA curves of some functional H-MOFs, for example, $\mathrm{H}-\mathrm{UiO}-$ 66- $\mathrm{NH}_{2}(\mathrm{Zr})$, exhibited a continuous weight loss similar to its

Table 1 | Pore features of eight representative H-MOFs (prepared by one-pot reaction) and their parent MOFs.

\begin{tabular}{|c|c|c|c|c|c|c|c|c|c|}
\hline H-MOF and MOF & $\begin{array}{l}\text { MOA template } \\
\text { precursor type }\end{array}$ & $\begin{array}{c}S_{\mathrm{BET}}^{\star} \\
\left(\mathrm{m}^{2} \mathrm{~g}^{-1}\right)\end{array}$ & $\begin{array}{c}S_{\text {micro }}^{\dagger} \\
\left(m^{2} g^{-1}\right)\end{array}$ & $S_{\text {micro }} / S_{\text {meso }} \ddagger$ & $\begin{array}{c}V_{t^{\S}}^{\S} \\
\left(m^{2} g^{-1}\right)\end{array}$ & $\begin{array}{c}V_{\text {micro }}{ }^{I I} \\
\left(m^{2} g^{-1}\right)\end{array}$ & $V_{\text {micro }} / V_{\text {meso }}$ & $\begin{array}{l}\text { Micro pore } \\
\text { size }(\AA)^{\#}\end{array}$ & $\begin{array}{l}\text { Meso por } \\
\text { size }^{\star \star}(\AA)\end{array}$ \\
\hline H-ZIF-8 & MOF-5 & 1,611 & 1,117 & 2.26 & 0.82 & 0.29 & 0.55 & 12.7 & 38.5 \\
\hline ZIF-8 & & 1,737 & 1,737 & & 0.7 & & & 11 & \\
\hline H-MIL-101(Cr) & ZIF-8 & 441 & 157 & 0.55 & 0.58 & 0.07 & 0.13 & 51 & 123 \\
\hline MIL-101(Cr) & & 2,927 & 2,357 & & 1.49 & & & $29 / 34$ & \\
\hline H-DUT-5 & In-BPDC & 1,183 & 737 & 1.65 & 0.91 & 0.68 & 2.96 & 11.0 & 39.4 \\
\hline DUT-5 & & 1,652 & 1,424 & & 0.82 & & & 11.1 & \\
\hline $\mathrm{H}-\mathrm{UiO}-66(\mathrm{Zr})$ & $\mathrm{Zn}_{4} \mathrm{O}(\mathrm{BC})_{6}$ & 917 & 300 & 0.49 & 0.87 & 0.13 & 0.17 & 11.8 & 38 \\
\hline $\mathrm{UiO}-66(\mathrm{Zr})$ & & 1,204 & 1,024 & & 0.59 & & & 8/11 & \\
\hline $\mathrm{H}-\mathrm{UiO}-66-\mathrm{NH}_{2}(\mathrm{Zr})$ & IRMOF-3 & 600 & 327 & 1.19 & 0.49 & 0.14 & 0.38 & $11.8 / 14.7$ & 56.2 \\
\hline $\mathrm{UiO}-66-\mathrm{NH}_{2}(\mathrm{Zr})$ & & 1,070 & 1,052 & & 0.42 & & & $7.4 / 9.5$ & \\
\hline $\mathrm{H}-\mathrm{UiO}-66-\mathrm{Cl}(\mathrm{Zr})$ & $\mathrm{Zn}_{4} \mathrm{O}(\mathrm{BC})_{6}$ & 758 & 334 & 0.79 & 0.81 & 0.15 & 0.23 & 12 & 56.2 \\
\hline UiO-66-CI(Zr) & & 794 & 750 & & 0.33 & & & $5.8 / 7.5$ & \\
\hline $\mathrm{H}-\mathrm{UiO}-66-\mathrm{Br}(\mathrm{Zr})$ & MOP-tBu & 558 & 221 & 0.66 & 0.50 & 0.11 & 0.29 & 11 & 55.5 \\
\hline $\mathrm{UiO}-66-\mathrm{Br}(\mathrm{Zr})$ & & 806 & 615 & & 0.43 & & & $5.6 / 7.3$ & \\
\hline $\mathrm{H}-\mathrm{UiO}-66(\mathrm{Hf})$ & MOP-tBu & 505 & 171 & 0.51 & 0.77 & 0.09 & 0.12 & 11.2 & 52.5 \\
\hline UiO-66(Hf) & & 890 & 739 & & 0.43 & & & $8 / 11$ & \\
\hline
\end{tabular}

H-MOF, hierarchical-pore MOF; MOA, metal-organic assembly; MOF, metal-organic framework

${ }^{*} S_{B E T}$ is the Brunauer-Emmett-Teller (BET) specific surface area.

$+S_{\text {miro }}$ is the $t$-plot-specific micropore surface area calculated from the $N_{2}$ adsorption-desorption isotherm.

$\pm S_{\text {meso }}$ is the specific mesopore surface area estimated by subtracting $S_{\text {micro }}$ from $S_{B E T}$

$\S V_{t}$ is the total specific pore volume determined by using the adsorption branch of the $N_{2}$ isotherm at $P / P_{0}=0.99$

$\| V_{\text {meso }}$ is the specific mesopore volume obtained from the Barrett-Joyner-Halenda (BJH) cumulative specific adsorption volume of pores of $1.70-300.00 \mathrm{~nm}$ in diameter.

T $V_{\text {micro }}$ is the specific micropore volume calculated by subtracting $V_{\text {meso }}$ from $V_{t}$.

\#The micropore diameter is determined by the density functional theory (DFT) method.

${ }^{\star \star}$ The mesopore diameter is determined from the local maximum of the $\mathrm{BJH}$ distribution of pore diameters obtained in the adsorption branch of the $\mathrm{N}_{2}$ isotherm at $77 \mathrm{~K}$. 
parent $\mathrm{UiO}-66-\mathrm{NH}_{2}(\mathrm{Zr})$ at the whole temperature range, which might be ascribed to the introduction of polar group in these MOFs (Supplementary Fig. 30) ${ }^{38}$.

In addition, we also noticed that the template not only can create mesopore for H-MOFs but also can affect the microporosity of the MOFs (Table 1). For example, the micropore surface area of $\mathrm{H}-\mathrm{UiO}-66(\mathrm{Zr})$ (prepared by using $\mathrm{Zn}_{4} \mathrm{O}(\mathrm{BC})_{6}$ precursors as the template source) is $300 \mathrm{~m}^{2} \mathrm{~g}^{-1}$, which is much smaller than that of parent UiO-66(Zr) $\left(1,024 \mathrm{~m}^{2} \mathrm{~g}^{-1}\right)$, whereas the micropore size is 11.8 and $14.1 \AA$, being larger than those in UiO-66(Zr) ( $8 \AA$ and $11 \AA$, corresponding to the tetrahedral and octahedral cages, respectively). For other H-MOFs, the similar results are observed. That is, the introduction of templates can decrease the micropore surface area and increase the micropore diameter at different degrees of final H-MOFs. In particular, for the H-MIL-101(Cr), there should have been various mesopores with the sizes across the two mesoporous cages ( 29 and $34 \AA$ ) in parent MIL-101(Cr). As a result, no step adsorption similar to that in MIL-101(Cr) was observed in H-MIL-101(Cr) (Supplementary Fig. 12j).

It should be pointed out that the development of reliable methods to rationally prepare $\mathrm{H}-\mathrm{MOF}$ with controllable/ tailorable structures and properties is much more challenged. In this work, we have definitely confirmed that the mesopore sizes of the resulting $\mathrm{H}-\mathrm{MOF}$ are tunable by varying the amount of the MOAs templates. Taking $\mathrm{H}-\mathrm{UiO}-66(\mathrm{Zr})$ as an example, Fig. 3 shows that the mesopore diameters of prepared materials can vary from 40 to $300 \AA$, depending on the amount of $\mathrm{Zn}_{4} \mathrm{O}(\mathrm{BC})_{6}$ or MOF-5 template precursors used in synthesis. It must be pointed out here that based on our experimental results, only when the amounts of $\mathrm{Zn}_{4} \mathrm{O}(\mathrm{BC})_{6}$ template precursors were controlled in the range of $0.25 \sim 0.75$ equiv., the decrease of template precursor amounts can increase the mesopore sizes in the resulting $\mathrm{H}-\mathrm{UiO}-66(\mathrm{Zr})$. As shown in Supplementary Figs 9 and 23 , the $\mathrm{H}-\mathrm{UiO}-66(\mathrm{Zr})$ became of poor crystallinity and even amorphous if the template precursors were more than 0.75 equiv., while no mesopore structure was created when less than 0.25 equiv. This observed relationship between mesopore size and the amount of template precursor may be related to the size of the formed template fragments under different precursor concentration conditions. In general, in crystallization process, a lower concentration of the precursor will lead to a lower nucleation rate, which results in a lower concentration of nuclei in the reaction system. A limited number of nuclei grow slowly in the reaction system, consequently resulting in bigger crystal particles. Otherwise, a higher concentration of the precursor can lead to a higher nucleation rate and much more nuclei, thus smaller crystal particles can be expected at a higher concentration of the precursor. As a consequence, an increase of the concentration (amount) of precursor in the reaction mixture usually resulted in a decrease of the generated template particle size in a given concentration range ${ }^{50}$. To further verify the tunability of mesoporosity of H-MOFs prepared by this approach, the mesopore sizes of H-ZIF-8, H-MIL-101(Cr) and H-DUT-5 synthesized with different amounts of template precursor were also tested (Supplementary Figs 13-15). It can be seen clearly from these results that the mesopore sizes of these H-MOFs are dependent on the amount of template precursors used in each case.

As stated above, the ligand-extension strategy for expanding pore size of MOFs often induces the interpenetration and instability of the MOF frameworks. Nevertheless, using our in situ self-assembly template method that takes the advantage of relative stability of different MOAs, stable mesoporous structures of H-MOFs can be easily created. Furthermore, the resulting $\mathrm{H}$-MOFs with tunable mesopore sizes are stable just similar to their parents, as confirmed in the case of $\mathrm{H}-\mathrm{UiO}-66(\mathrm{Zr})$ and UiO-66(Zr) (Supplementary Fig. 43).

Large-molecule adsorption in H-MOFs. We know that adsorbents with large pores could be used in the capture of large

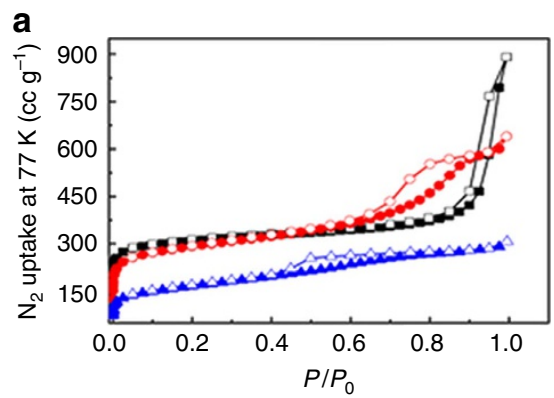

C

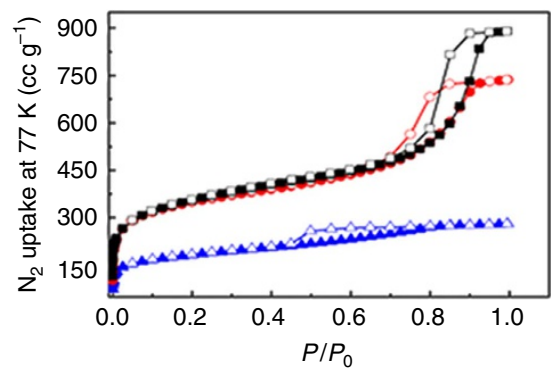

b

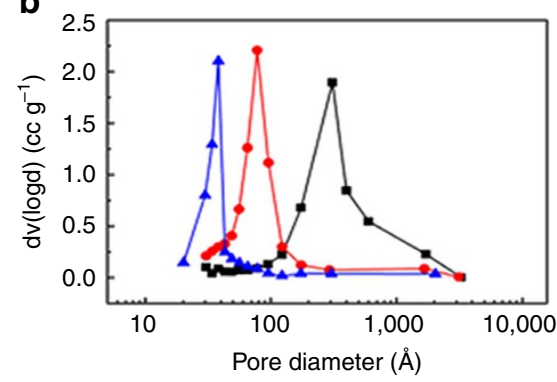

d

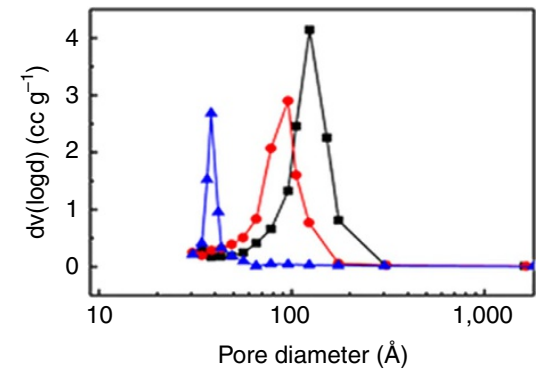

Figure 3 | Tuning the mesopore size through changing the amount of template. $(\mathbf{a}, \mathbf{c}) \mathrm{N}_{2}$ adsorption-desorption isotherms at $77 \mathrm{~K}$ and $(\mathbf{b}, \mathbf{d})$ pore size distributions of $\mathrm{H}-\mathrm{UiO}-66(\mathrm{Zr})$ prepared with different amounts of template (preparation conditions: (a,b) $1 \mathrm{ml}$ (black curve), $2 \mathrm{ml}$ (red curve) and $3 \mathrm{ml}$ (blue curve) of nanosized MOF-5 suspension solution in DMF as the template precursor (the MOF- 5 concentration in the suspension solution is about $12 \mathrm{mg} \mathrm{ml}^{-1}$ ); (c,d) 0.25 equiv. (black curve), 0.375 equiv. (red curve) and 0.5 equiv. (blue curve) of $\mathrm{Zn}_{4} \mathrm{O}(\mathrm{BC}$ ) 6 template precursor (equiv. means the equivalent of $\mathrm{Zn}_{4} \mathrm{O}(\mathrm{BC})_{6}$ with respect to $\left.\mathrm{ZrCl}_{4}\right)$ ). 

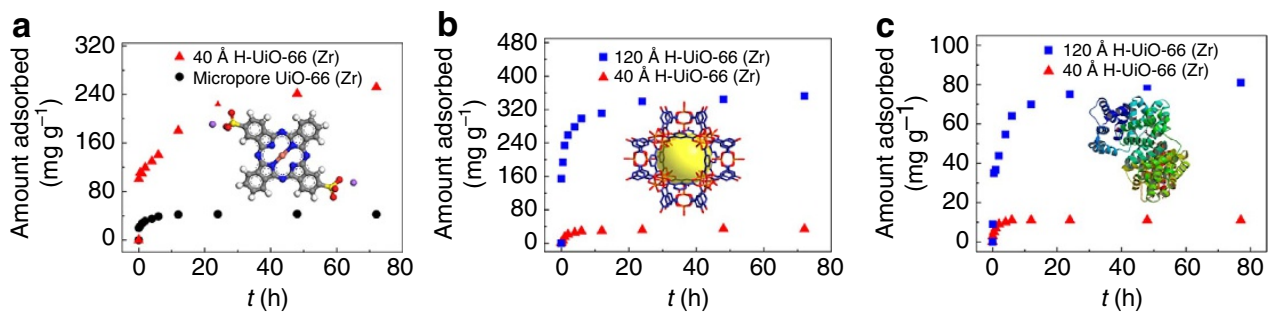

Figure 4 | Large-molecule adsorption in H-UiO-66(Zr). (a) Adsorption kinetics of dye DB 86 in microporous UiO-66( $\mathrm{Zr}$ ) and 40 $\AA$ (means mesopore size in the H-MOF) H-UiO-66( $\mathrm{Zr}$ ), (b) adsorption kinetics of MOP-OH in 40 and $120 \AA \mathrm{H}-\mathrm{UiO}-66(\mathrm{Zr})$, and (c) adsorption kinetics of BSA in 40 and $120 \AA$ $\mathrm{H}-\mathrm{UiO}-66(\mathrm{Zr})$.

molecules. Here, taking $\mathrm{H}-\mathrm{UiO}-66(\mathrm{Zr})$ as a representative of prepared H-MOFs in this work, we performed liquid-phase adsorption experiments to explore the accessibility of their mesopores towards large molecules with different sizes. For this purpose, organic dye molecule (Direct Blue 86, DB 86, about $4 \times 12 \times 14 \AA$ in size), MOPs (MOP-OH, about $40 \times 40 \times 40 \AA$ in size) and biological protein molecule (bovine serum albumin (BSA), about $140 \times 40 \times 40 \AA$ in size) were selected as the probe molecules. As shown in Fig. 4, microporous UiO-66(Zr) almost can not adsorb DB 86 molecules from aqueous solution, but H-UiO-66(Zr) with about $40 \AA$ mesopores can efficiently capture this large molecule. With regard to recognizing different mesopore sizes of $\mathrm{H}-\mathrm{HiO}-66(\mathrm{Zr})$, larger MOP-OH and BSA were further checked. It was found that the $\mathrm{H}-\mathrm{UiO}-66(\mathrm{Zr})$ with $40 \AA$ mesopores can hardly accommodate the two types of molecules, but the one with $120 \AA$ mesopores can. Although the length of BAS is a little bit longer than the mesopore size of the H-UiO$66(\mathrm{Zr})$, this material can still accommodate the protein, which can be attributed to the slender geometry of BAS and the irregular shape of mesopore in it. In addition, white $\mathrm{H}-\mathrm{UiO}-66(\mathrm{Zr})$ powder exhibited a colour change after DB 86 or MOP-OH adsorption as shown in Supplementary Fig. 44. Clearly, these stable H-MOFs with easily tuned mesopores can find potential applications in large-molecule adsorption/separation.

\section{Discussion}

Foregoing results demonstrate that using an in situ self-assembly template method, by taking advantage of relative stability of different metal-organic coordination assemblies, stable H-MOFs with tailorable pore sizes can be easily prepared based on their stable parent microporous MOFs. Although the exact nature of the template can not be identified at this stage, this approach is quite feasible and seems to be versatile in preparing H-MOFs. Such types of hierarchical-pore materials may provide promising applications in large-molecule adsorption/separation, heterogeneous catalysis and drug release due to their several distinct merits, such as having co-existed micropores and mesopores, large pore size, good stability and improved mass transfer in their pores.

\section{Methods}

Synthesis. Experimental procedures for preparing all the H-MOFs achieved in this work are provided in the Supplementary Information. Here we take H-UiO$66(\mathrm{Zr})$ preparation with MOF-5 precursor as the template source, as an example to describe the preparation method. The synthesis started by mixing parent MOF precursors and template precursors, including $0.120 \mathrm{~g}$ of $\mathrm{ZrCl}_{4}, 0.160 \mathrm{~g}$ of $\mathrm{H}_{2} \mathrm{BDC}$, $0.297 \mathrm{~g}$ of $\mathrm{Zn}\left(\mathrm{NO}_{3}\right)_{2} \cdot 6 \mathrm{H}_{2} \mathrm{O}$, with $20 \mathrm{ml}$ of DMF in a 100 -ml Teflon liner. After sonication for $10 \mathrm{~min}$ the Teflon liner vessel was sealed and placed in a preheated oven at $120^{\circ} \mathrm{C}$ for $24 \mathrm{~h}$. After cooling to room temperature, the resulted powder was separated by centrifugation. The supernatant was discarded and the solid was washed several times with DMF to remove the unreacted precursors, to give asprepared template@H-UiO-66(Zr). To remove the acid-sensitive MOA templates, the solid was dispersed in $10 \mathrm{ml}$ of diluted $\mathrm{HCl}$ solution $(\mathrm{pH} 1)$ and stirred for about $10 \mathrm{~min}$. After discarding the supernatant, the obtained solid was re-dispersed in DMF and then centrifuged. This process was repeated three times so that all decomposed template fragments were removed. Next, a similar process was performed by using acetone as the solvent, instead of DMF, afterwhich the solid was heated at $150^{\circ} \mathrm{C}$ for $12 \mathrm{~h}$ at vacuum, to get activated sample for $\mathrm{N}_{2}$ adsorption measurement.

Characterization. The characterizations of MOF-5 particle size, EA, ICP, SEM, transmission electron microscope, $\mathrm{N}_{2}$ adsorption-desorption isotherms at $77 \mathrm{~K}$, PXRD, TGA, FT-IR, XPS and NMR spectra are given in Supplementary Information. Particle size distributions and SEM micrograph of MOF-5 particles are shown in Supplementary Figs 1 and 2. $\mathrm{N}_{2}$ adsorption-desorption isotherms and pore size distributions of H-MOFs are included in Supplementary Figs 3-15. PXRD patterns of H-MOFs are given in Supplementary Figs 16-23. TGA curves of H-MOFs are given in Supplementary Figs 24-30. FT-IR spectra of H-MOFs are given in Supplementary Figs 31-35. XPS spectra of H-MOFs are included in Supplementary Figs 36 and $37 .{ }^{13} \mathrm{C}-\mathrm{NMR}$ spectra of $\mathrm{H}-\mathrm{UiO}-66(\mathrm{Zr})$ are given in Supplementary Figs 38 and 39. HAADF-STEM image and corresponding elemental maps in template@H-UiO-66(Zr) are shown in Supplementary Figs 40-42. PXRD patterns of $\mathrm{H}-\mathrm{UiO}-66(\mathrm{Zr})$ before and after soaking in water for $24 \mathrm{~h}$ are given in Supplementary Fig. 43. Photographs of H-UiO-66(Zr) before and after large molecular adsorption are shown in Supplementary Fig. 44. Porosity properties of various H-MOFs and their parent MOFs are included in Supplementary Table 1. Element mole ratios in template@H-MOFs and H-MOFs are given in Supplementary Table 2 .

\section{References}

1. Bruce, D. W., O., Hare, D. \& Walton, R. I. Porous Materials (Wiley, 2010).

2. Zhou, H.-C., Long, J. R. \& Yaghi, O. M. Introduction to metal-organic frameworks. Chem. Rev. 112, 673-674 (2012).

3. Long, J. R. \& Yaghi, O. M. The pervasive chemistry of metal-organic frameworks. Chem. Soc. Rev. 38, 1213-1214 (2009).

4. Sumida, K. et al. Carbon dioxide capture in metal-organic frameworks. Chem Rev. 112, 724-781 (2012).

5. Li, J.-R., Sculley, J. \& Zhou, H.-C. Metal-organic frameworks for separations. Chem. Rev. 112, 869-932 (2012).

6. Yang, Q., Liu, D., Zhong, C. \& Li, J. -R. Development of computational methodologies for metal-organic frameworks and their application in gas separations. Chem. Rev. 113, 8261-8323 (2013).

7. Cavka, J. H. et al. A new zirconium inorganic building brick forming metal-organic frameworks with exceptional stability. J. Am. Chem. Soc. 130, 13850-13851 (2008).

8. Park, K. S. et al. Exceptional chemical and thermal stability of zeolitic imidazolate frameworks. Proc. Natl Acad. Sci. USA 103, 10186-10191 (2006).

9. Loiseau, T. et al. A rationale for the large breathing of the porous aluminum terephthalate (MIL-53) upon hydration. Chem. Eur. J. 10, 1373-13823 (2004)

10. Xuan, W., Zhu, C., Liu, Y. \& Cui, Y. Mesoporous metal-organic framework materials. Chem. Soc. Rev. 41, 1677-1695 (2012).

11 . Feng, D. et al. A highly stable zeotype mesoporous zirconium metal-organic framework with ultralarge pores. Angew. Chem. Int. Ed. 54, 149-154 (2015).

12. Wang, K. et al. A series of highly stable mesoporous metalloporphyrin Fe-MOFs. J. Am. Chem. Soc. 136, 13983-13986 (2014).

13. Senkovska, I. \& Kaskel, S. Ultrahigh porosity in mesoporous MOFs: promises and limitations. Chem. Commun. 50, 7089-7098 (2014).

14. Wang, T. C. et al. Ultrahigh surface area zirconium MOFs and insights into the applicability of the BET theory. J. Am. Chem. Soc. 137, 3585-3591 (2015).

15. Song, L. et al. Mesoporous metal-organic frameworks: design and applications. Energy Environ. Sci. 5, 7508-7520 (2012).

16. Eddaoudi, M. et al. Systematic design of pore size and functionality in isoreticular MOFs and their application in methane storage. Science 295, 469-472 (2002). 
17. Deng, H. et al. Large-pore apertures in a series of metal-organic frameworks. Science 336, 1018-1023 (2012).

18. Farha, O. K. et al. Metal-organic framework materials with ultrahigh surface areas: is the sky the limit. J. Am. Chem. Soc. 134, 15016-15021 (2012).

19. Yan, Y., Yang, S., Blake, A. J. \& Schröder, M. Studies on metal-organic frameworks of $\mathrm{Cu}(\mathrm{II})$ with isophthalate linkers for hydrogen storage. Acc. Chem. Res. 47, 296-307 (2014).

20. Qiu, L. et al. Hierarchically micro- and meso-porous metal-organic frameworks with tunable porosity. Angew. Chem. Int. Ed. 47, 9487-9491 (2008).

21. Feng, S. et al. Synthesis of nitrogen-doped hollow carbon nanospheres for $\mathrm{CO}_{2}$ capture. Chem. Commun. 50, 329-331 (2014).

22. Zhao, Y. et al. Metal-organic framework nanospheres with well-ordered mesopores synthesized in an ionic liquid/ $/ \mathrm{CO}_{2} /$ surfactant system. Angew. Chem. Int. Ed. 50, 636-639 (2011).

23. Wee, L. H. et al. Copper benzene tricarboxylate metal-organic framework with wide permanent mesopores stabilized by keggin polyoxometallate ions. J. Am. Chem. Soc. 134, 10911-10919 (2012).

24. Sun, L., Li, J. -R., Park, J. \& Zhou, H. -C. Cooperative template-directed assembly of mesoporous metal-organic frameworks. J. Am. Chem. Soc. 134, 126-129 (2012)

25. Peng, L. et al. Surfactant-directed assembly of mesoporous metal-organic framework nanoplates in ionic liquids. Chem. Commun. 48, 8688-8690 (2012).

26. $\mathrm{Wu}$, Y. et al. Amino acid assisted templating synthesis of hierarchical zeolitic imidazolate framework-8 for efficient arsenate removal. Nanoscale 6, 1105-1112 (2014).

27. Pham, M., Vuong, G., Fontaine, F. \& Do, T. A route to bimodal micromesoporous metal-organic frameworks nanocrystals. Cryst. Growth. Des. 12, 1008-1013 (2012).

28. Do, X., Hoang, V. \& Kaliaguine, S. MIL-53(Al) mesostructured metal-organic frameworks. Micropor. Mesopor. Mat. 141, 135-139 (2011).

29. Cao, S. et al. Hierarchical bicontinuous porosity in metal-organic frameworks templated from functional block co-oligomer micelles. Chem. Sci. 4, 3573-3577 (2013).

30. Ma, T. et al. Ordered mesoporous metal-organic frameworks consisting of metal disulfonates. Chem. Mater. 24, 2253-2255 (2012).

31. Xue, Z. et al. Poly(ethylene glycol) stabilized mesoporous metal-organic framework nanocrystals: efficient and durable catalysts for the oxidation of benzyl Alcohol. ChemPhysChem 15, 85-89 (2014).

32. Li, L. et al. A synthetic route to ultralight hierarchically micro/mesoporous Al(III)-carboxylate metal-organic aerogels. Nat. Commun. 4, 1774-1783 (2013).

33. Peng, L. et al. Highly mesoporous metal-organic framework assembled in a switchable solvent. Nat. Commun. 5, 4456-4463 (2014).

34. Reboul, J. et al. Mesoscopic architectures of porous coordination polymers fabricated by pseudomorphic replication. Nat. Mater. 11, 717-723 (2012).

35. Morris, R. E. \& Čejka, J. Exploiting chemically selective weakness in solids as a route to new porous materials. Nat. Chem. 7, 381-388 (2015).

36. Schröck, K., Schröder, F., Heyden, M., Fischer, R. A. \& Havenith, M. Characterization of interfacial water in MOF-5 $\left(\mathrm{Zn}_{4}(\mathrm{O})(\mathrm{BDC})_{3}\right)$, a combined spectroscopic and theoretical study. Phys. Chem. Chem. Phys. 10, 4732-4739 (2008).

37. Greathouse, J. A. \& Allendorf, M. D. The interaction of water with MOF-5 simulated by molecular dynamics. J. Am. Chem. Soc. 128, 10678-10679 (2006).

38. DeCoste, J. B. et al. Stability and degradation mechanisms of metal-organic frameworks containing the $\mathrm{Zr}_{6} \mathrm{O}_{4}(\mathrm{OH})_{4}$ secondary building unit. J. Mater. Chem. A 1, 5642-5650 (2013).

39. Han, Y., Li, J.-R., Xie, Y. \& Guo, G. Substitution reactions in metal-organic frameworks and metal-organic polyhedra. Chem. Soc. Rev. 43, 5952-5981 (2014).

40. Deria, P. et al. Beyond post-synthesis modification: evolution of metal-organic frameworks via building block replacement. Chem. Soc. Rev. 43, 5896-5912 (2014).
41. Abney, C. W. et al. Topotactic transformations of metal-organic frameworks to highly porous and stable inorganic sorbents for efficient radionuclide sequestration. Chem. Mater. 26, 5231-5243 (2014).

42. Perry, J. T., Perman, J. A. \& Zaworotko, M. J. Design and synthesis of metal-organic frameworks using metal-organic polyhedra as supermolecular building blocks. Chem. Soc. Rev. 38, 1400-1417 (2009).

43. Li, J. -R., Timmons, D. J. \& Zhou, H. -C. Interconversion between molecular polyhedra and metal-organic frameworks. J. Am. Chem. Soc. 131, 6368-6369 (2009).

44. Li, J. -R. \& Zhou, H. -C. Bridging-ligand-substitution strategy for the preparation of metal-organic polyhedra. Nat. Chem. 2, 893-898 (2010).

45. Kim, M. et al. Postsynthetic ligand and cation exchange in robust metal-organic frameworks. J. Am. Chem. Soc. 134, 18082-18088 (2012).

46. Karagiaridi, O. et al. Opening ZIF-8: a catalytically active zeolitic imidazolate framework of sodalite topology with unsubstituted linkers. J. Am. Chem. Soc. 134, 18790-18796 (2012)

47. Schaate, A. et al. Modulated synthesis of Zr-based metal-organic frameworks: from nano to single crystals. Chem. Eur. J. 17, 6643-6651 (2011).

48. $\mathrm{Wu}, \mathrm{H}$. et al. Unusual and highly tunable missing-linker defects in zirconium metal-organic framework UiO-66 and their important effects on gas adsorption. J. Am. Chem. Soc. 135, 10525-10532 (2013).

49. Vermoortele, F. et al. Synthesis modulation as a tool to increase the catalytic activity of metal-organic frameworks: the unique case of UiO-66(Zr). J. Am. Chem. Soc. 135, 11465-11468 (2013).

50. Shevchenko, E. V. et al. Study of nucleation and growth in the organometallic synthesis of magnetic alloy nanocrystals: the role of nucleation rate in size control of $\mathrm{CoPt}_{3}$ nanocrystals. J. Am. Chem. Soc. 125, 9090-9101 (2003).

\section{Acknowledgements}

This work is supported by the National Key Basic Research Program of China ('973') (number 2013CB733503), the Natural Science Foundation of China (numbers 21322601, 21271015, 21136001 and 21322603), the Program for New Century Excellent Talents in University (numbers NCET-13-0647 and NCET-12-0755) and the Beijing Municipal Natural Science Foundation (number 2132013).

\section{Author contributions}

J.R.L., C.Z. and H.H. conceived and designed the experiments, and co-wrote the paper. H.H. performed most of experiments and analysed data. L.L. and K.W. participated in the $\mathrm{N}_{2}$ adsorption test. T.H. and M.T. performed the liquid adsorption experiment under the guidance of Q.Y. and D.L. Y.X. performed the PXRD and TGA test. All authors discussed the results and commented on the manuscript.

\section{Additional information}

Supplementary Information accompanies this paper at http://www.nature.com/ naturecommunication

Competing financial interests: The authors declare no competing financial interests.

Reprints and permission information is available online at http://npg.nature.com/ reprintsandpermissions/

How to cite this article: Huang, H. et al. An in situ self-assembly template strategy for the preparation of hierarchical-pore MOFs. Nat. Commun. 6:8847 doi: $10.1038 /$ ncomms 9847 (2015)

This work is licensed under a Creative Commons Attribution 4.0 International License. The images or other third party material in this article are included in the article's Creative Commons license, unless indicated otherwise in the credit line; if the material is not included under the Creative Commons license, users will need to obtain permission from the license holder to reproduce the material. To view a copy of this license, visit http://creativecommons.org/licenses/by/4.0/ 